\title{
Rocaglamide overcomes tumor necrosis factor-related apoptosis-inducing ligand resistance in hepatocellular carcinoma cells by attenuating the inhibition of caspase-8 through cellular FLICE-like-inhibitory protein downregulation
}

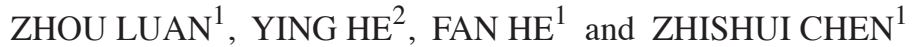 \\ ${ }^{1}$ Institute of Organ Transplantation, Tongji Hospital, Tongji Medical College, Huazhong University of Science and Technology, \\ Wuhan, Hubei 430030; ${ }^{2}$ Department of Ophthalmology, The Central Hospital of Wuhan, \\ Wuhan, Hubei 430014, P.R. China
}

Received October 18, 2013; Accepted September 4, 2014

DOI: $10.3892 / \mathrm{mmr} .2014 .2718$

\begin{abstract}
The enhancement of apoptosis is a therapeutic strategy used in the treatment of cancer. Tumor necrosis factor-related apoptosis-inducing ligand (TRAIL) is a promising antitumor agent. However, hepatocellular carcinoma (HCC) cells exhibit marked resistance to the induction of cell death by TRAIL. The present study investigated whether rocaglamide, a naturally occurring product isolated from the genus Aglaia, is able to sensitize resistant HCC cells to TRAIL-mediated apoptosis. Two HCC cell lines, HepG2 and Huh-7, were treated with rocaglamide and/or TRAIL and the induction of apoptosis and effects on the TRAIL signaling pathway were investigated. The in vivo efficacy of rocaglamide was determined in TRAIL-resistant Huh-7-derived tumor xenografts. Rocaglamide significantly sensitized the TRAIL-resistant HCC cells to apoptosis by TRAIL, which resulted from the rocaglamide-mediated downregulation of cellular FLICE-like inhibitory protein and subsequent caspase- 8 activation. Furthermore, rocaglamide markedly inhibited tumor growth from Huh-7 cells propagated in severe combined immunodeficient mice, suggesting that chemosentization also occurred in vivo. These data suggest that rocaglamide acted synergistically with TRAIL against the TRAIL-resistant HCC cells. Thus, it is concluded that rocaglamide as an adjuvant to TRAIL-based therapy may present a promising therapeutic approach for the treatment of HCC.
\end{abstract}

Correspondence to: Dr Zhishui Chen, Institute of Organ Transplantation, Tongji Hospital, Tongji Medical College, Huazhong University of Science and Technology, 1095 Jiefang Road, Wuhan, Hubei 430030, P.R. China

E-mail: zschen@tjh.tjmu.edu.cn

Key words: rocaglamide, tumor necrosis factor-related apoptosis-inducing ligand, hepatocellular carcinoma, cellular FLICE-like inhibitory protein, caspase-8

\section{Introduction}

Hepatocellular carcinoma (HCC) is one of the most common and life-threatening types of human malignant tumor (1). The inadequate effects of conventional chemotherapy and resistance to drugs present a challenge to HCC treatment. In particular, advanced HCC cells respond poorly to the induction of apoptosis by chemotherapeutic agents due to reprogramming of cellular apoptotic machinery (2). Thus, there is a requirement to examine potential targets of the cellular apoptotic machinery to develop novel and potent therapeutic drugs for the treatment of HCC.

Tumor necrosis factor-related apoptosis-induced ligand (TRAIL), a type II transmembrane protein from the tumor necrosis factor family, triggers the extrinsic pathway of apoptosis by binding to the cell surface receptors death receptor (DR) 4 and 5 (3). These receptors then form homomeric and heteromeric complexes and stimulate the recruitment of Fas-associated death domain (FADD) and caspase-8, which self-activate and initiate downstream caspase cleavage events (4). TRAIL is promising as a novel therapeutic agent due to its high potential for the selective induction of apoptosis (5). However, several studies have indicated that HCC cells are relatively refractory to TRAIL and that TRAIL alone is unable to induce apoptotic cell death in these cells (6). A number of factors may be responsible for the resistance of HCC cells to TRAIL. TRAIL-induced apoptosis is mediated by caspase- 8 (7) and the resistance of HCC cells to TRAIL is correlated with a downregulation in the activity of caspase-8, which disturbs apoptotic signals in the cancer cells (8). Notably, the activity of caspase- 8 is inhibited by its inhibitory protein, the cellular FLICE-like inhibitory protein (c-FLIP) in chemoresistant cells (9). c-FLIP is expressed at higher levels in TRAIL-resistant HCC cells compared with TRAIL-sensitive cells (10). Furthermore, the downregulation of c-FLIP renders highly resistant HCC cells sensitive to TRAIL treatment (11). Therefore, novel therapeutic strategies to eliminate the c-FLIP-mediated chemoresistance of HCC cells are required, and the identification of novel therapeutic compounds with 
anti-HCC activity, particularly those derived from naturally occurring materials, is necessary.

Rocaglamide is isolated from the genus Aglaia (family Meliaceae) (12). A number of species from this genus are used in traditional medicine to treat coughs, injuries, asthma and inflammatory skin diseases. Rocaglamide has also been observed to possess anticancer properties in leukemia $(13,14)$. However, the detailed mechanisms underlying the anticancer activities of rocaglamide in solid tumors remain to be elucidated.

The aim of the present study was to investigate whether rocaglamide sensitized resistant HCC cells to TRAIL-induced death, by regulation of caspase-8/c-FLIP in vitro. Furthermore, the efficacy of rocaglamide in TRAIL-resistant Huh-7-derived tumor xenografts was determined.

\section{Materials and methods}

Cell culture and reagents. HepG2 and Huh-7 cells were obtained from the Shanghai Cell Collection (Shanghai, China) and cultured in Dulbecco's modified Eagle's medium (DMEM; Gibco-BRL, Carlsbad, CA, USA) supplemented with $10 \%$ fetal bovine serum (FBS; Gibco-BRL), $2 \mathrm{mM}$ glutamine, $100 \mathrm{U} / \mathrm{ml}$ penicillin and $100 \mu \mathrm{g} / \mathrm{ml}$ streptomycin. The cells were cultured at $37^{\circ} \mathrm{C}$ in $5 \% \mathrm{CO}_{2}$. Rocaglamide ( $>98 \%$ pure) was procured from Enzo Life Sciences (Lörrach, Germany). TRAIL was purchased from PeproTech, Inc. (Rocky Hill, NJ, USA) and all chemicals were purchased from Sigma (St. Louis, MO, USA), unless indicated otherwise.

Treatment of cells with rocaglamide and/or TRAIL. For the investigation of time-dependence, the cells (70\% confluent) were treated with rocaglamide $(100 \mathrm{nM})$ and/or TRAIL $(100 \mathrm{ng} / \mathrm{ml})$ for different time periods $(0-24 \mathrm{~h})$. The cells were then harvested for cell viability analysis. For the investigation of dose-dependence, the cells (70\% confluent) were pretreated with rocaglamide (0-100 $\mathrm{nM})$ for $12 \mathrm{~h}$ in DMEM, supplemented with $10 \%$ FBS, 2 mM glutamine, $100 \mathrm{U} / \mathrm{ml}$ penicillin and $100 \mu \mathrm{g} / \mathrm{ml}$ streptomycin, and were then treated with TRAIL (0-100 ng/ml) for an additional $12 \mathrm{~h}$. To investigate treatment with a combination of rocaglamide and TRAIL, the cells were pretreated with either rocaglamide or dimethyl sulfoxide (DMSO) for $12 \mathrm{~h}$, followed by $12 \mathrm{~h}$ incubation in the presence of TRAIL. Subsequently, $24 \mathrm{~h}$ after rocaglamide or DMSO treatment, the cells were harvested and the cell lysates were prepared, according to previous methods (15) and stored at $-80^{\circ} \mathrm{C}$ for later use.

Cell viability assay. HepG2 and Huh-7 cells (1x104/well) were seeded in 96-well plates in complete culture medium and incubated for $24 \mathrm{~h}$. The cells were then exposed to $100 \mathrm{nM}$ rocaglamide and/or $100 \mathrm{ng} / \mathrm{ml}$ TRAIL for $24 \mathrm{~h}$. The control cells were treated with DMSO at a concentration equal to that used for the drug-treated cells. The complete culture medium was then removed and MTT $(200 \mu 1,0.5 \mathrm{mg} / \mathrm{ml}$ in $10 \%$ FBS-containing DMEM) was added to each well and the plate was incubated for $2 \mathrm{~h}$ at $37^{\circ} \mathrm{C}$ in a humidified incubator. The solution was then removed from the wells and $200 \mu$ 1 DMSO was added to each well prior to agitation. The absorbance at $570 \mathrm{~nm}$ was read using a microplate reader (Bio-Tek ELx800; BioTek Instruments Inc., Winooski, VT, USA). The value for the vehicle-treated cells was considered to indicate $100 \%$ viability. Furthermore, a crystal violet assay was carried out. Briefly, the cells $\left(1.0 \times 10^{5} / \mathrm{ml}\right)$ were seeded in a 12-well plate for $12 \mathrm{~h}$, and treated with TRAIL $(0-100 \mathrm{ng} / \mathrm{ml})$ and/or RocA(1-100 $\mathrm{nM})$ for $12 \mathrm{~h}$. The treated cells were washed with phosphate-buffered saline (PBS), fixed with $4 \%$ paraformaldehyde for $15 \mathrm{~min}$, and stained using crystal violet (Sigma; cat no. C3886) for a further $30 \mathrm{~min}$.

Western blot analysis. Western blot analysis was performed, as described previously (7) using the following: mouse anti-c-FLIP monoclonal antibody (NF6; cat.no ALX-804-428; Alexis Biochemicals, San Diego, CA, USA), mouse anti-caspase-8 monoclonal antibody (cat.no 9746; Cell Signaling Technology, Inc. Danvers, MA, USA), mouse anti-DR4 monoclonal antibody (cat.no ab13890; Abcam, Cambridge, UK), rabbit anti-DR5 polyclonal antibody (cat. no ab47179; Abcam), Pro-Apoptosis Bcl-2 Family Antibody Sampler kit (cat.no 9942; Cell Signaling Technology, Inc.) and Apoptosis Western Blot Cocktail (cat.no ab136812; Abcam). An enhanced chemiluminescence detection reagent (SuperSignal West Pico) was used for detection (Pierce Biotechnologies, Rockford, IL, USA) and $\alpha$-tubulin and $\beta$-actin were used as loading controls. All western blots were representative of at least three independent experiments.

Transfection of c-FLIP. Small interfering (si)RNA control or siRNA FLIP (Santa Cruz Biotechnology) of high purity were delivered into the HepG2 cells in the 12-well plate using Lipofectamine 2000 (Invitrogen Life Technologies, Carlsbad, CA, USA) according to the manufacturer's instructions. Briefly, $2 \mu 1$ Lipofectamine 2000 was added to $50 \mathrm{nmol} / 1$ siRNA in a final volume of $100 \mu \mathrm{l}$ culture medium and the solution was added to the washed cells, which were then incubated at $37^{\circ} \mathrm{C}$ for $4 \mathrm{~h}$. The transfection mixture was then removed and the cells were incubated with fresh complete medium for an additional 36 h. Finally, the cells were exposed to TRAIL or DMSO for $12 \mathrm{~h}$ for subsequent experiments.

Detection of apoptosis and necrosis using flow cytometry. The HepG2 and Huh-7 cells were seeded and cultured in each well of a 12-well plate for $24 \mathrm{~h}$ and then pretreated with either rocaglamide or DMSO for $12 \mathrm{~h}$, followed by $12 \mathrm{~h}$ incubation in the presence of TRAIL. An annexin V/propidium iodide (PI) apoptosis detection kit (eBioscience, Inc., San Diego, CA, USA) was used for the detection of apoptotic and necrotic cells. The cells $\left(5 \times 10^{5}\right)$ were harvested and resuspended in $400 \mu \mathrm{l} 1 \mathrm{X}$ binding buffer with $4 \mu \mathrm{l}$ FITC annexin $\mathrm{V}$ and $4 \mu \mathrm{l}$ PI. Flow cytometric analysis was then performed using a Becton-Dickinson FACSCalibur ${ }^{\mathrm{TM}}$ system; BD Biosciences, Franklin Lakes, NJ, USA) and FlowJo 7.6.3 software (Tree Star, Inc., Ashland, OR, USA).

Tumorigenicity studies in severe combined immunodeficient (SCID) mice. The Huh-7 cells $\left(3 \times 10^{6}\right)$, suspended in $100 \mu 1 \mathrm{mix}$ (equal volumes of DMEM and Matrigel), were implanted subcutaneously into the right flank of 10 female SCID mice (6-week-old) and then randomly divided into two equal groups, one of which received an intraperitoneal injection of rocaglamide $(2.5 \mathrm{mg} / \mathrm{kg}$ in $80 \mu \mathrm{l}$ olive oil; $\mathrm{n}=5)$ and the other, used as a vehicle control, received olive oil alone 
$(n=5)$. These treatments were performed once daily for 32 days and the tumor volumes and body weights of the animals were measured twice a week. The tumor volumes $\left(\mathrm{mm}^{3}\right)$ were calculated using the following formula: Tumor volume $=\mathrm{LS}^{2} / 2$, where $\mathrm{L}$ is the longest diameter and $\mathrm{S}$ is the shortest. At the end of the experiments, the mice were sacrificed and tumor samples were harvested, fixed in formalin and embedded in paraffin as tissue sections for immunohistochemical analysis. The animal experiments were performed in accordance with the relevant institutional and national regulations and the research procedures were approved by Tongji Medical College (Wuhan, China). The SCID mice were purchased from Beijing HFK Bioscience, Co., Ltd. (Beijing, China).

Immunohistochemistry and terminal deoxynucleotidyltransferase-mediated dUTP nick end labeling (TUNEL) staining. Immunohistochemical staining was performed using either 1:1 hematoxylin and $0.5 \%$ eosin $(\mathrm{H} \& \mathrm{E})$ or an anti-cleaved caspase-3 antibody (Cell Signaling Technology, Inc.), as previously described (16). Apoptosis was assessed by TUNEL (In situ Cell Death Detection kit; Roche Diagnostics, Basel, Switzerland) according to the manufacturer's instructions. The primary antibody was omitted in the first step to establish a negative control, which yielded negative results. The percentage of positive cells was independently determined by two examiners. At least five random fields of each section were visualized (magnification $\mathrm{x} 400$ ) and analyzed using a Nikon OPTIPHOT 150 microscope (Nikon, Tokyo, Japan) connected to a SPOT Insight charge-coupled device camera (Diagnostic Instruments, Inc., Sterling Heights, MI, USA). The values are expressed as the mean \pm standard deviation (SD).

Statistical analysis. Where indicated, the data are expressed as the mean \pm SD from at least three independent experiments. Statistical analysis of the data was conducted using Student's t-test. $\mathrm{P}<0.01$ was considered to indicate a statistically significant difference.

\section{Results}

Rocaglamide enhances TRAIL-induced apoptosis in resistant $H C C$ cells. To investigate whether rocaglamide enhanced TRAIL-mediated apoptosis in HCC cells in the present study, HepG2 and Huh-7 cells, which are highly chemoresistant to TRAIL (17), were selected. The effect of rocaglamide on TRAIL-induced cytotoxicity was then examined using an MTT assay. Treatment with rocaglamide or TRAIL alone was minimally cytotoxic to the HepG2 and Huh-7 cells; however, pretreatment of the cells with rocaglamide enhanced the cytotoxic effect of TRAIL in a time-dependent manner in the two cell lines (Fig. 1B). Furthermore, the effect of rocaglamide on TRAIL-induced cell death was demonstrated by observing the morphological signs of apoptosis. Although rocaglamide and TRAIL alone did not induce morphological signs of cell death, rocaglamide markedly enhanced the effect of TRAIL-induced apoptosis (Fig. 1C). Cell apoptosis was also confirmed using annexin V/PI staining and flow cytometry in the HepG2 and Huh-7 cells. Treatment with rocaglamide alone led to apoptosis in $\sim 9 \%$ HepG2 and $11 \%$ Huh-7 cells and treatment with TRAIL induced apoptosis in $\sim 16 \%$ HepG2 and $17 \%$
Huh-7 cells. However, the combination of rocaglamide and TRAIL induced apoptosis in $~ 55 \%$ HepG2 and 57\% Huh-7 cells (Fig. 1D and E), which is evidently more than an additive effect. A similar result was obtained by measurement of cell viability using crystal violet staining (Fig. 1F). Taken together, the data from the present study indicate that rocaglamide has the potential to sensitize highly chemoresistant HepG2 and Huh-7 cells to TRAIL-based therapy.

Rocaglamide promotes TRAIL-induced caspase-dependent apoptotic cell death. TRAIL-induced apoptosis is mediated by activation of the caspase cascade (18). In particular, the cleavage of caspase- 8 is an essential step in the TRAIL-mediated caspase activation cascade (19). Therefore, the present study investigated whether the cleavage of caspase- 8 was triggered in TRAIL-resistant cell lines following treatment with rocaglamide or TRAIL alone. The results revealed that modest reductions in the level of the procaspase- 8 protein occurred in the rocaglamide-treated and TRAIL-treated HepG2 and Huh-7 cells. An increase in the level of active-caspase- 8 was also observed in these cells (Fig. 2A; lanes 2 and 3 vs. lane 1). However, combined treatment with rocaglamide and TRAIL significantly augmented the TRAIL-induced cleavage/activation of caspases- 8 (Fig. 2A; lane 4 vs. lanes 1, 2 and 3). Notably, treatment with rocaglamide alone did not affect the expression levels of pro-caspase- 3 compared with the control (Fig. 2B; lane 2 vs. lane 1). Furthermore, treatment with TRAIL alone resulted in a small reduction in the level of pro-caspase-3 and a small increase in the cleavage of poly ADP ribose polymerase (PARP) and activated caspase-3 substrates in the HepG2 and Huh-7 cells (Fig. 2B; lane 3 vs. lanes 1 and 2). However, combined treatment with rocaglamide and TRAIL resulted in significantly increased activity of the TRAIL-induced cleavage/activation of pro-caspase-3 and cleavage of PARP (Fig. 2B; lane 4 vs. lanes 1,2 and 3), indicating that combined treatment induced apoptotic death in the hepG2 and Huh-7 cells, at least partly through a caspase-dependent pathway. Thus, these results clearly suggest that rocaglamide sensitizes TRAIL-resistant HCC cells to TRAIL-induced cell death through the enhancement of caspase activity.

As pro-apoptotic B-cell lymphoma 2 (Bcl-2) family proteins, including $\mathrm{BH}$-interacting domain death agonist (BID), Bcl-2-associated X protein (BAX) and p53-upregulated modulator of apoptosis (PUMA), are able to sensitize cancer cells to TRAIL-induced apoptosis (20), the present study investigated the expression levels of these proteins. In the HepG2 and Huh-7 cells treated with the rocaglamide/TRAIL combination, the protein levels of pro-apoptotic Bcl-2-interacting mediator of cell death (BIM), Bcl-2 homologous antagonist killer (BAK) and PUMA were significantly increased, whereas the protein levels of other pro-apoptotic proteins, including BID and BAX were reduced (Fig. 2C), indicating the possible inhibition of protein synthesis by rocaglamide (21). Therefore, these results suggest that the upregulation of the BIM, BAK and PUMA proteins is associated with the rocaglamide-mediated sensitization of HepG2 and Huh-7 cells to TRAIL-induced apoptosis. Overall, these results indicate that rocaglamide substantially increases the apoptotic potential of TRAIL in HCC cells through extrinsic and intrinsic pathways. 
A<smiles>COc1ccc(C2(c3ccc(OC)cc3OC)c3ccccc3N(C)C(=O)[C@H]2O)cc1</smiles>

B

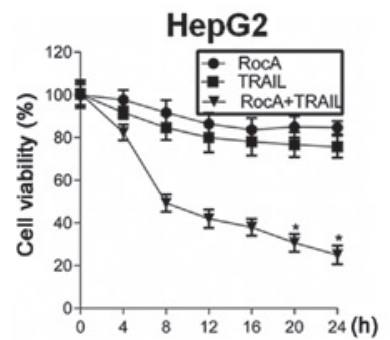

Huh-7

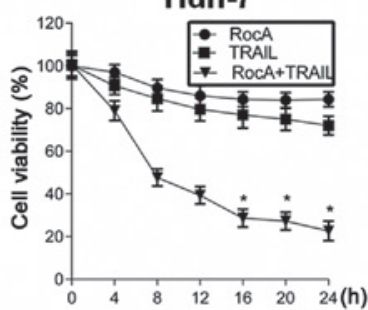

C

HepG2

DMSO

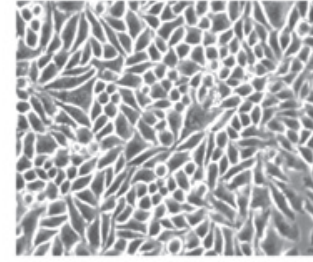

RocA

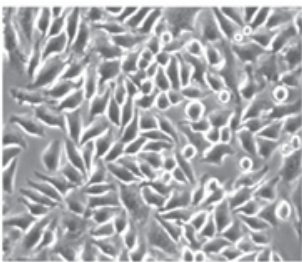

TRAIL

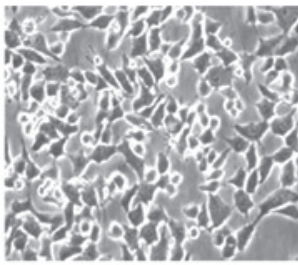

TRAIL
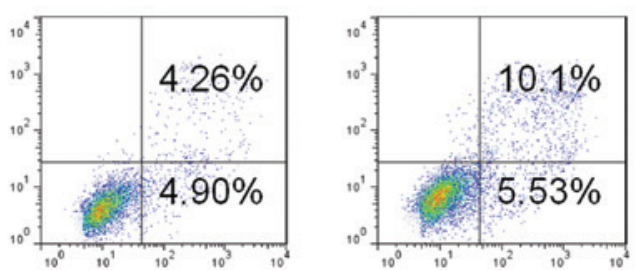

RocA+TRAIL

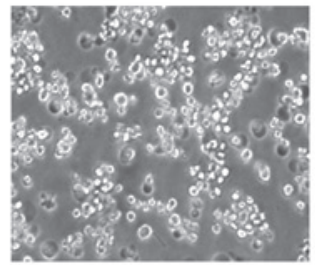

D

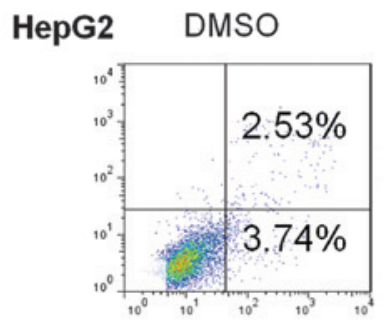

Huh-7

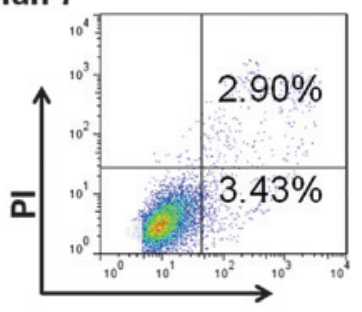

Annexin V
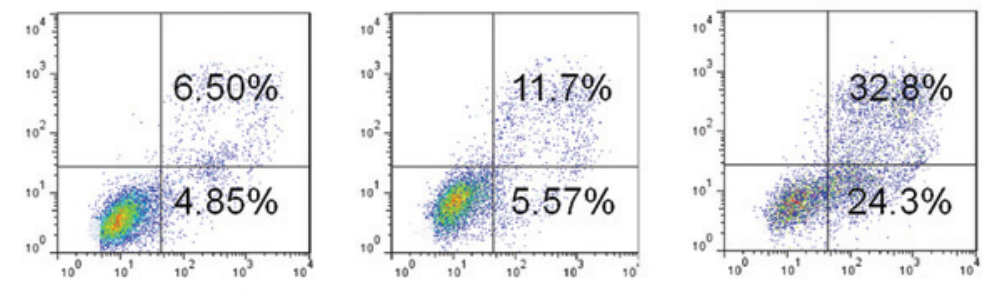

TRAIL + RocA

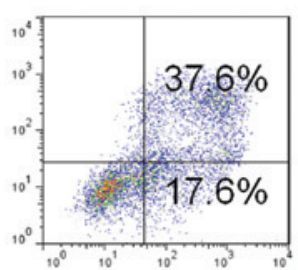

E

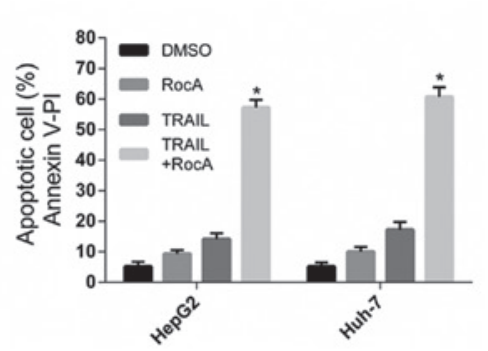

F

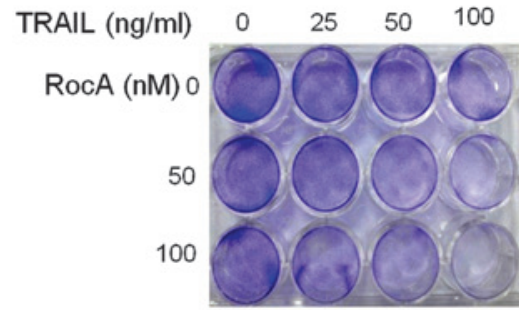

HepG2

Figure 1. RocA sensitizes HCC cells to TRAIL-mediated apoptosis. (A) Chemical structure of RocA. (B) HepG2 and Huh-7 cells were treated with RocA $(100 \mathrm{nM})$ and/or TRAIL $(100 \mathrm{ng} / \mathrm{ml})$ for different time periods, as indicated. Cell viability was measured using an MTT assay. (C) Representative images of the cells were captured using phase-contrast microscopy (magnification, x400). (D and E) HepG2 and Huh-7 cells were pretreated with RocA (100 nM) for $12 \mathrm{~h}$ and then exposed to TRAIL $(100 \mathrm{ng} / \mathrm{ml})$ for $12 \mathrm{~h}$. Cell apoptosis was determined using flow cytometry with annexin/PI double staining. (F) Cells were washed with PBS and fixed with $4 \%$ paraformaldehyde and were stained using crystal violet. Data are expressed as the mean \pm standard deviation. $\mathrm{P}<0.01$, TRAIL+RocA group vs TRAIL group. Data are representative of three experiments. RocA, rocaglamide; HCC, hepatocellular carcinoma; TRAIL, tumor necrosis factor-related apoptosis-inducing ligand; DMSO, dimethyl sulfoxide; PI, propidium iodide.

Rocaglamide sensitizes TRAIL-induced apoptosis via c-FLIP downregulation. c-FLIP, which is highly homologous to caspase- 8 but is catalytically inactive, is able to bind to caspase- 8 and the FADD and inhibit TRAIL-induced apoptosis, thus disrupting the death-inducing signaling complex (DISC) (22). Notably, HCC tumors exhibit high resistance to
TRAIL due to the overexpression of c-FLIP (23). Following the observation that rocaglamide is able to overcome TRAIL-resistance in the HCC cells and activate caspase- 8 , the present study investigated whether this effect correlated with c-FLIP. Therefore, the effect of rocaglamide on the levels of c-FLIP, which is highly expressed in HCC cells, was examined 


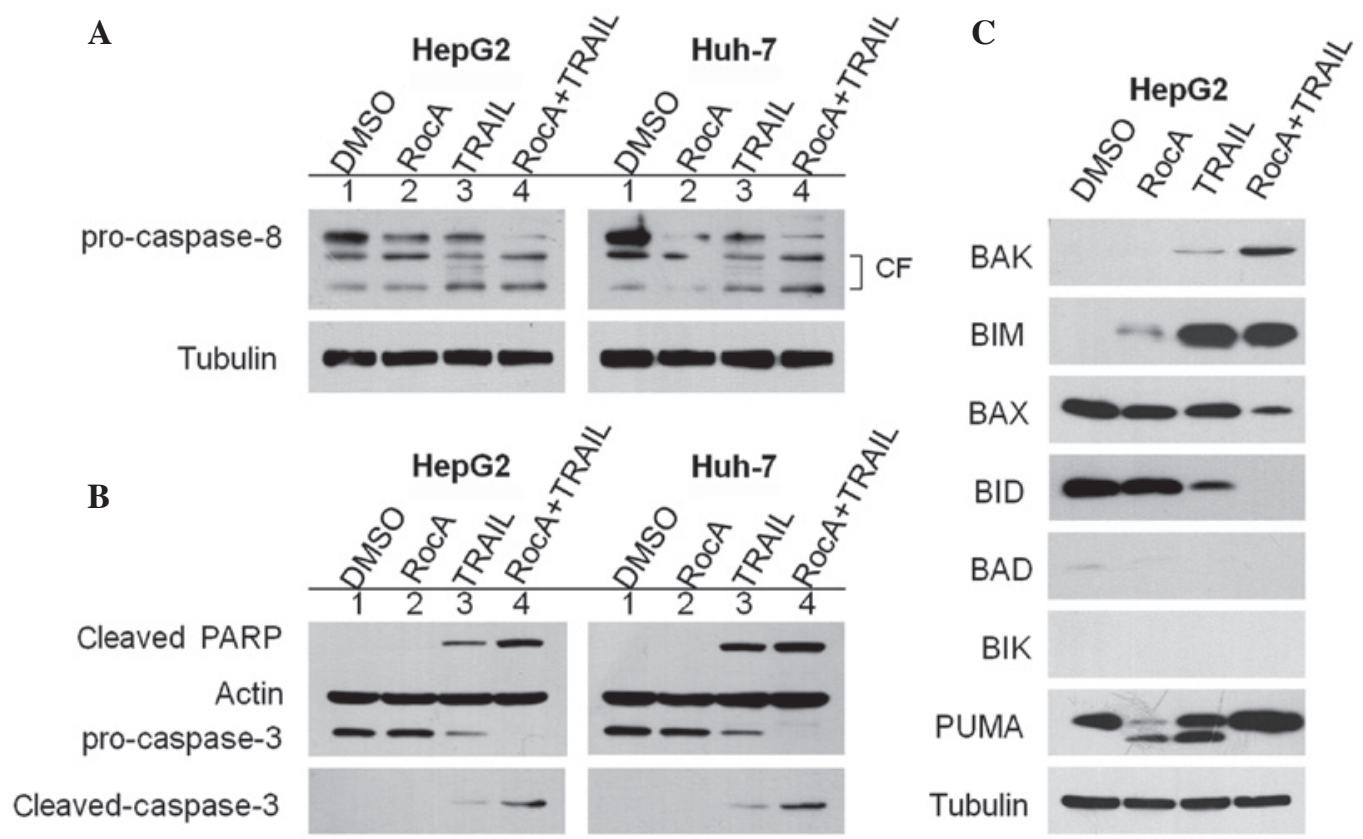

Figure 2. RocA enhances caspase activity triggered by TRAIL. (A and B) HepG2 and Huh-7 cells were pretreated with RocA (100 nM) for $12 \mathrm{~h}$ and then exposed to TRAIL $(100 \mathrm{ng} / \mathrm{ml})$ for $12 \mathrm{~h}$. Cell lysates were subjected to western blot analysis using the antibodies indicated. (C) HepG2 cells were pretreated with RocA $(100 \mathrm{nM})$ for $12 \mathrm{~h}$ and TRAIL $(100 \mathrm{ng} / \mathrm{ml})$ for $12 \mathrm{~h}$. The cells were lysed and western blot analysis was performed using the antibodies indicated. The control contained cells treated with DMSO only. Results are representative of three independent experiments. RocA, rocaglamide; CF, cleaved (active) form; TRAIL, tumor necrosis factor-related apoptosis-inducing ligand; DMSO, dimethyl sulfoxide; PARP, poly ADP ribose polymerase; BAK, Bcl-2 homologous antagonist killer; BIM, Bcl-2-interacting mediator of cell death; BAX, Bcl-2-associated X protein; BID, BH3-interacting domain death agonist; BAD, Bcl-2-associated death promoter; BIK, Bcl-2-interacting killer; PUMA, p53-upregulated modulator of apoptosis.

A

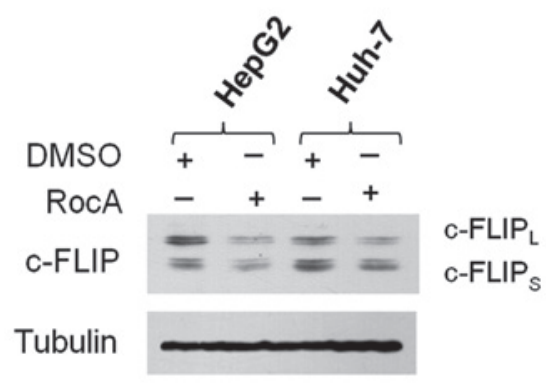

B

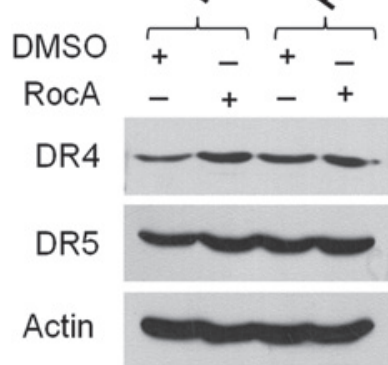

Figure 3. RocA downregulates c-FLIP. (A and B) HepG2 and Huh-7 cells were treated with RocA (100 nM) for 12 h. The cell lysates were analyzed using western blot analysis with the antibodies indicated. The control is represented by cells treated with DMSO only. Results are representative of three independent experiments. RocA, rocaglamide; DMSO, dimethyl sulfoxide; c-FLIP, cellular FLICE-like inhibitory protein; DR, death receptor.

(Fig. 3A). Notably, the resulting data also suggest that rocaglamide treatment resulted in downregulation of the c-FLIP protein in the HepG2 and Huh-7 cells (Fig. 3A). The resulting effect of rocaglamide on the levels of c-FLIP was consistent with the effects of rocaglamide on caspase-dependent apoptosis.

TRAIL triggers the extrinsic pathway of apoptosis by binding to cognate DR4 or DR5 (3), which results in the formation of receptor homotrimers and the propagation of a proapoptotic signal through caspase-8, which then self-activates and initiates downstream caspase events (24). In addition, the levels of DR4 or DR5 correlate with the sensitivity of TRAIL-mediated cell death (25). Therefore, the present study investigated the effect of rocaglamide on DR4 and DR5. No significant changes in the levels of DR4 or DR5 were observed in the rocaglamide-treated HepG2 and Huh-7 cells (Fig. 3B).

Knockdown of c-FLIP mimics the effect of rocaglamide in overcoming TRAIL-resistance in HepG2 cells. In order to determine the effect of downregulation of c-FLIP on the rocaglamide-induced effect, the present study also examined whether the apoptosis-inducing effects of rocaglamide were also exerted in c-FLIP-knockdown HepG2 cells. siRNA was used to specifically downregulate c-FLIP and an siRNA vector served as a control. This treatment resulted in a marked suppression in the levels of c-FLIP of $\sim 70 \%$ (Fig. 4A). The cell viability, evaluated by crystal violet staining, indicated that the c-FLIP-silenced HepG2 cells exhibited an increased sensitivity to TRAIL treatment compared with the that of the 
A

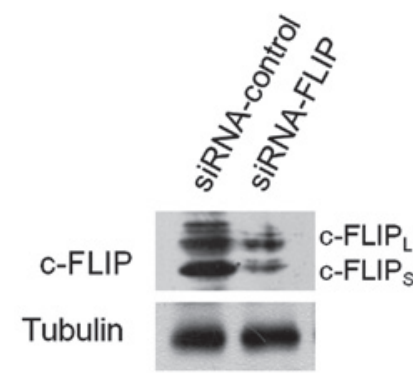

D

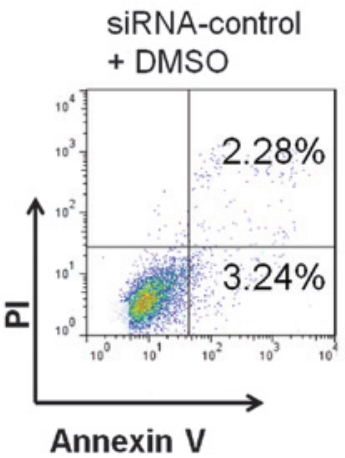

B

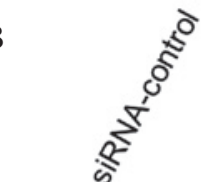
S

TRAIL
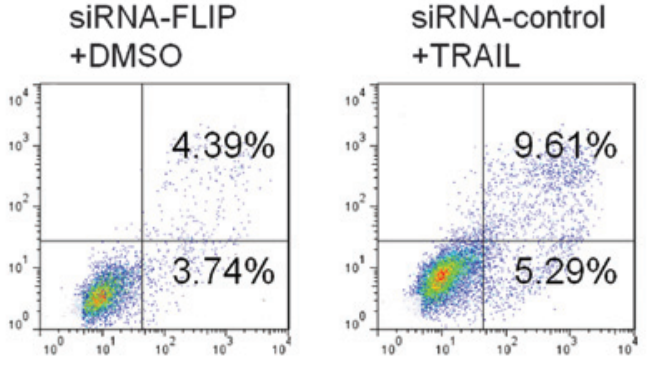
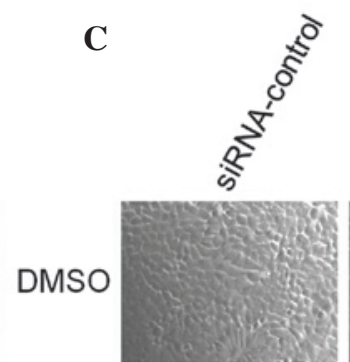

TRAIL

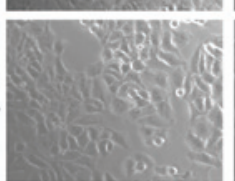

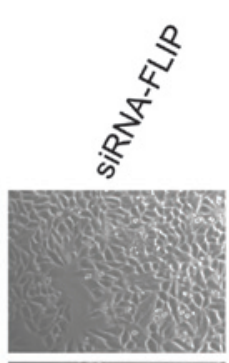
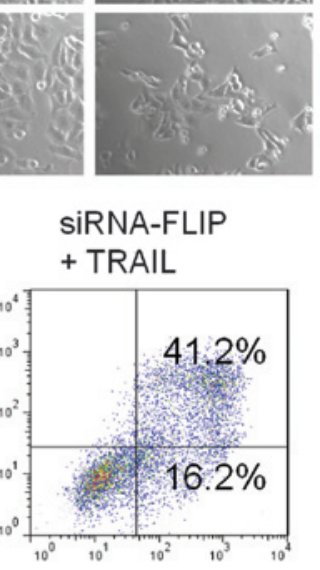

$\mathbf{E}$

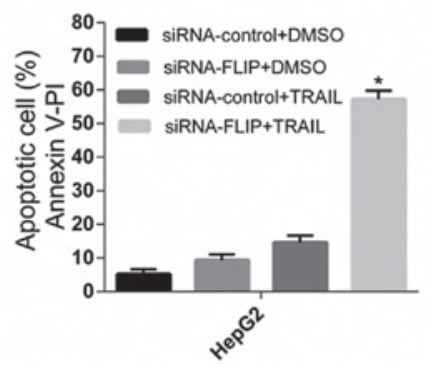

Figure 4. Knockdown of c-FLIP mimics the effect of rocaglamide in sensitizing HepG2 cells to TRAIL. The HepG2 cells were transiently transfected with either siRNA specific for c-FLIP or a control for $48 \mathrm{~h}$ and were then treated with TRAIL (100 ng/ml) for $12 \mathrm{~h}$. (A) Western blot analysis indicating the expression levels of c-FLIP in the c-FLIP-silenced and control cells. (B) Cells were washed with PBS and fixed with 4\% paraformaldehyde followed by staining using crystal violet. (C) Representative images of c-FLIP-silenced HepG2 and control cells. (D and E) Cell apoptosis was determined using flow cytometry with annexin/PI double staining in the c-FLIP-knockdown HepG2 and control cells (magnification, x400). Data are expressed as the mean \pm standard deviation. ${ }^{*} \mathrm{P}<0.01$, siRNA-FLIP+TRAIL group vs siRNA-control+TRAIL group. Results are representative of three independent experiments. TRAIL, tumor necrosis factor-related apoptosis-inducing ligand; DMSO, dimethyl sulfoxide; c-FLIP, cellular FLICE-like inhibitory protein; PI, propidium iodide.

control (Fig 4B). In addition, the morphological signs of cell death were significantly increased following treatment with TRAIL alone in the c-FLIP-silenced HepG2 cells (Fig. 4C). Furthermore, apoptosis was analyzed using annexin V/PI staining by flow cytometry in the c-FLIP-knockdown HepG2 cells. The results revealed that the induced rate of apoptosis was $\sim 8 \%$ by siRNA-FLIP, $15 \%$ by TRAIL and $56 \%$ by siRNA-FLIP combined with TRAIL (Fig. 4D and E), suggesting that the downregulation of c-FLIP in the HepG2 cells mimicked the effect of rocaglamide on TRAIL-mediated apoptosis in the TRAIL-resistant HCC cells.

Rocaglamide induces apoptosis of tumor cells in SCID mice models. To confirm whether the synergistic effect of rocaglamide and TRAIL in resistant cell lines had potentially relevant clinical implications, the present study investigated the in vivo effect of rocaglamide and TRAIL on the growth of HCC xenograft tumors. As natural killer cells produce TRAIL in vivo $(26,27)$, rocaglamide alone was administered in the in vivo study. The Huh-7 cells were subcutaneously injected into the right flanks of SCID mice and, when tumors were visible, the mice were matched for tumor volumes and were assigned to a control group and a rocaglamide-treated group. Tumor volumes in the rocaglamide-treated group were $\sim 45 \pm 12 \%$ compared with the control group (Fig. 5A). Rocaglamide significantly suppressed tumor growth compared with that in the control group. Notably, treatment with rocaglamide did not lead to any reduction in body weight and no apparent signs of toxicity were observed in the mice during the treatment (Fig. 5B), suggesting that rocaglamide is generally tolerated well in vivo.

The present study further investigated the effect of rocaglamide on apoptosis in vivo by examining tumor tissues harvested from the control and rocaglamide-treated mice 
A

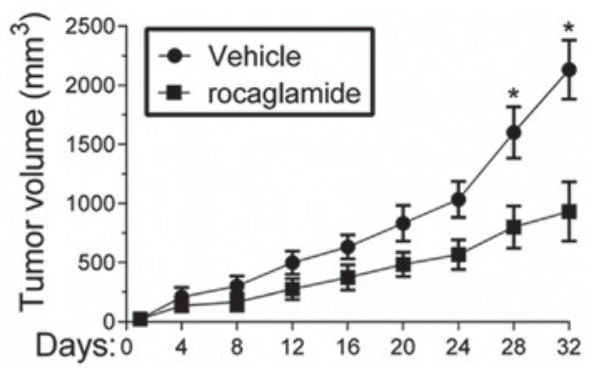

$\mathbf{C}$

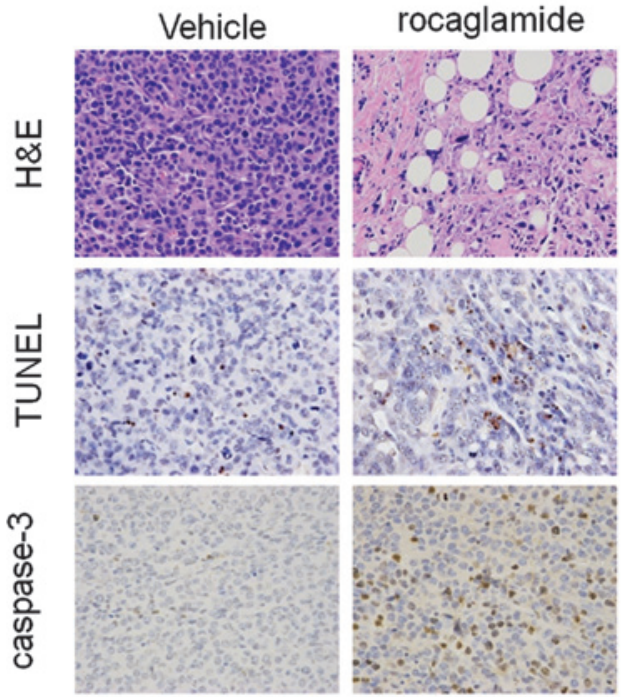

B

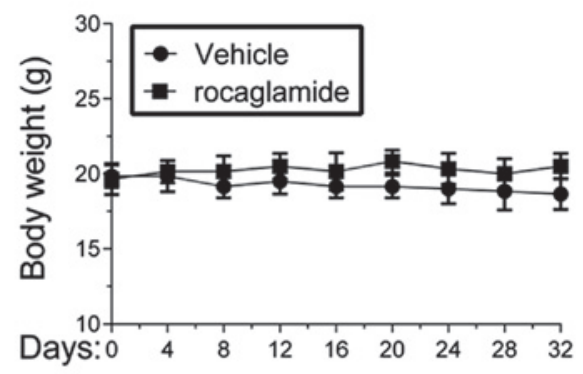

$\mathbf{D}$
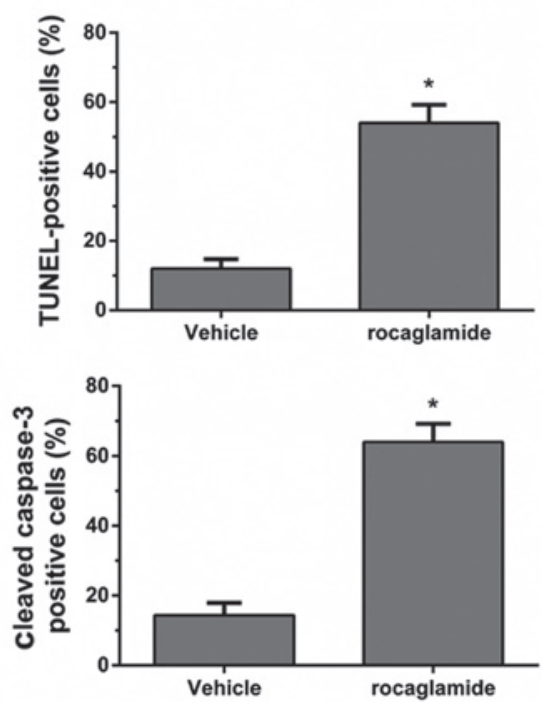

Figure 5. Effect of rocaglamide treatment on the growth of tumors from Huh-7 cells implanted in SCID mice. Huh-7 cell tumors established subcutaneously in SCID mice were treated with vehicle (5\% DMSO in olive oil; $\mathrm{n}=5)$ or rocaglamide $(2.5 \mathrm{mg} / \mathrm{kg} ; \mathrm{n}=5)$ via daily intraperitoneal injection into the mice for 32 days. (A) Tumor volume and (B) body weight were monitored. (C) Histological analysis of apoptosis in xenografted HCC tumors from the mice were performed using H\&E, TUNEL and cleaved caspase-3 staining (magnification, x200). (D) Quantitation of the TUNEL and cleaved caspase-3 staining in rocaglamide-treated tumors compared with vehicle-treated tumors. Data are expressed as the mean \pm standard deviation. "P<0.01 compared with vehicle. Data are representative of three experiments. SCID, severe combined immunodeficient; DMSO, dimethyl sulfoxide; H\&E, hematoxylin and eosin; TUNEL, terminal deoxynucleotidyltransferase-mediated dUTP nick end labeling.

using H\&E, TUNEL and cleaved caspase-3 staining. In the untreated controls, staining with H\&E revealed a compact mass of epithelial cells, whereas following rocaglamide treatment, the tumor appearance was that of loose epithelial cell aggregates with increased interspersed mesenchymal cells (Fig. 5C, top panels). In addition, the TUNEL assays demonstrated a three-fold increase in the percentage of apoptotic cells in the rocaglamide treatment group compared with the untreated controls (Fig. 5C, middle panels and D, upper panel). Furthermore, the cleaved caspase-3 staining confirmed a five-fold increase in apoptosis in the tumor sections from the group treated with rocaglamide, relative to the untreated control (Fig. 5C, bottom panels and D, lower panel). Therefore, the in vivo investigation suggests that rocaglamide is an effective drug, which has the potential to inhibit the growth of HCC cell-derived tumors in vivo.

\section{Discussion}

HCC is a significant cause of mortality worldwide due to its poor prognosis (28). The ability to evade apoptosis is a characteristic property of HCC cells, which results mainly from the lack of response to apoptotic stimuli (29). HCC cells have acquired drug resistance to cell death and are frequently refractory to classical chemotherapy (30). Thus, new strategies are required to address the resistance of HCC to apoptosis in order to improve the poor prognosis. Considerable attention has been directed towards investigating the effect of triggering apoptosis in HCC cells using natural products that stimulate DR-mediated apoptosis (31). In the present study, rocaglamide, a naturally occurring product, was demonstrated to sensitize TRAIL-resistant HCC cells to apoptosis through the suppression of c-FLIP in both in vitro and in vivo conditions.

As the type of cancer cell in HCC may affect the response of the cell to therapeutic agents, the robustly TRAIL-resistant HCC cell lines, HepG2 and Huh-7, were selected (16). Furthermore, the effect of rocaglamide on HepG2 and Huh-7 cells was examined, as was their sensitivity to TRAIL. Notably, the HepG2 and Huh-7 cells, were minimally responsive to treatment with TRAIL alone, indicating complete resistance to TRAIL. Treatment of the TRAIL-resistant HepG2 and Huh-7 cells with rocaglamide resulted in dose-dependent growth inhibition. Thus, these data provide evidence that rocaglamide has the potential to inhibit the proliferation of HCC cells and lead to their elimination.

TRAIL-based therapy offers promising therapeutic potential due to its specificity for cancer cells without evident adverse effects on normal cells $(5,32)$. However, resistance 
to TRAIL-mediated therapy has been observed in HCC cells, which indicates that treatment with TRAIL alone may be ineffective in treating HCC. A noteworthy observation from the present study is that the HepG2 and Huh-7 cells, which are highly TRAIL-resistant, increased in sensitivity to TRAIL following pretreatment with rocaglamide, supporting the possibility of investigating rocaglamide as an adjuvant to combination therapy for HCC patients. In addition, rocaglamide triggered the process of apoptosis in the presence of TRAIL at nanomolar concentrations, suggesting the efficacy of rocaglamide may be potent and non-toxic.

The detailed mechanisms of resistance to TRAIL in HCC cells remain to be elucidated. The regulation of DISC-engaged molecules, including c-FLIP and caspase- 8 , has been observed to contribute to the sensitivity of TRAIL-mediated apoptosis in cancer cells $(9,33,34)$. In addition, TRAIL resistance correlates with accelerated degradation of caspase- 8 protein in cancer cells $(8,35)$. The present study demonstrated that rocaglamide significantly activates caspase- 8 in $\mathrm{HCC}$ cells. Furthermore, the rocaglamide-induced caspase- 8 activation was found to be accompanied by increased cleavage of the apoptotic marker for caspase-3, PARP, in vitro. These findings are consistent with previous studies indicating that the activation of caspanse- 8 induces apoptosis and sensitizes cancer cells to TRAIL $(36,37)$.

c-FLIP inhibits the apoptotic signaling cascade by preventing the recruitment and activation of caspase- 8 at the DISC (38), demonstrating that an elevated intracellular level of c-FLIP confers resistance against proapoptotic stimuli in tumor cells (39). In addition, ectopic expression of c-FLIP inhibits the release of active caspase- 8 fragments from the DISC, resulting in disruption of the DISC complex (40). By contrast, downregulation of c-FLIP results in the sensitization of chemoresistant tumor cells (41). Collectively, these findings suggest that c-FLIP may be a novel therapeutic drug target for HCC. The present study provides evidence indicating that treatment with rocaglamide significantly reduces the protein expression level of c-FLIP in HepG 2 and Huh-7 cells at nanomolar concentrations. Furthermore, combined treatment with rocaglamide and TRAIL significantly induced apoptosis, suggesting that rocaglamide induces sensitivity to TRAIL by the suppression of c-FLIP in HepG2 cells. Notably, siRNA was used to downregulate c-FLIP in the HepG2 cells and revealed that the downregulation of c-FLIP in HepG2 cells mimicked the effect of rocaglamide on TRAIL-mediated apoptosis in TRAIL-resistant HCC cells.

Targeting the transcriptional activation of c-FLIP is considered to be a promising approach for the downregulation of c-FLIP expression in cancer cells (42). However, agents directly inhibiting FLIP at the mRNA and protein levels remain to be elucidated. Subsequent studies are planned to determine whether rocaglamide treatment attenuates the transcriptional activation of c-FLIP are required, and to examine whether the effect of rocaglamide on c-FLIP occurs at the transcriptional level. Consistent with previous studies, the results of the present study suggest that the downregulation of the transcriptional activation of c-FLIP sensitizes HCC cells to TRAIL. However, the detailed mechanisms involved in regulating the expression of c-FLIP remain to be elucidated.
The present study demonstrated that rocaglamide, a naturally occurring product, sensitized chemoresistant HCC cells to TRAIL-mediated apoptosis by decreasing the expression of c-FLIP and activating caspase- 8 in vitro. Furthermore, rocaglamide markedly inhibited the growth of tumors derived from Huh-7 cells in vivo in a xenograft mice model. Thus, these findings provide significant evidence for the development of rocaglamide as a novel therapeutic agent for use as an adjuvant to TRAIL in the treatment of HCC.

\section{Acknowledgements}

The present study wa supported by the National Natural Science Foundation of China (grant nos. 81370581, 81000290, 30972796).

\section{References}

1. Arzumanyan A, Reis HM and Feitelson MA: Pathogenic mechanisms in HBV- and HCV-associated hepathocellular carcinoma. Nat Rev Cancer 13: 123-135, 2013.

2. Wang K and Lin B: Inhibitor of apoptosis proteins (IAPs) as regulatory factors of hepatic apoptosis. Cell Signal 25: 1970-1980, 2013.

3. Kischkel FC, Lawrence DA, Chuntharapai A, Schow P, Kim KJ and Ashkenazi A: Apo2L/TRAIL-dependent recruitment of endogenous FADD and caspase- 8 to death receptors 4 and 5 . Immunity 12: 611-620, 2000.

4. Reed JC: Apoptosis-targeted therapies for cancer. Cancer Cell 3: 17-22, 2003.

5. Hall MA and Cleveland JL: Clearing the TRAIL for cancer therapy. Cancer Cell 12: 4-6, 2007.

6. Herr I, Schemmer P and Büchler MW: On the TRAIL to therapeutic intervention in liver disease. Hepatology 46: 266-274, 2007.

7. Dickens LS, Boyd RS, Jukes-Jones R, Hughes MA, Robinson GL, Fairall L, Schwabe JW, Cain K and Macfarlane M: A death effector domain chain DISC model reveals a crucial role for caspase-8 chain assembly in mediating apoptotic cell death. Mol Cell 47: 291-305, 2012.

8. Zhang L, Zhu H, Teraishi F, Davis JJ, Guo W, Fan Z and Fang B: Accelerated degradation of caspase-8 protein correlates with TRAIL resistance in a DLD1 human colon cancer line. Neoplasia 7: 594-602, 2005.

9. Haag C, Stadel D, Zhou S, Bachem MG, Möller P, Debatin KM and Fulda S: Identification of c-FLIP(L) and c-FLIP(S) as critical regulators of death receptor-induced apoptosis in pancreatic cancer cells. Gut 60: 225-237, 2011.

10. Du X, Bao G, He X, Zhao H, Yu F, Qiao Q, Lu J and Ma Q: Expression and biological significance of c-FLIP in human hepatocellular carcinoma. J Exp Clin Cancer Res 28: 24, 2009.

11. Lai LJ and Ho TC: Pigment epithelial-derived factor inhibits c-FLIP expression and assists ciglitazone-induced apoptosis in hepatocellular carcinoma. Anticancer Res 31: 1173-1180, 2011.

12. Kim S, Salim AA, Swanson SM and Kinghorn AD: Potential of cyclopenta[b]benzofurans from Aglaia species in cancer chemotherapy. Anticancer Agents Med Chem 6: 319-345, 2006.

13. Lucas DM, Edwards RB, Lozanski G, West DA, Shin JD, Vargo MA, Davis ME, Rozewski DM, Johnson AJ, Su BN, et al: The novel plant-derived agent silvestrol has B-cell selective activity in chronic lymphocytic leukemia and acute lymphoblastic leukemia in vitro and in vivo. Blood 113: 4656-4666, 2009.

14. Giaisi M, Köhler R, Fulda S, Krammer PH and Li-Weber M: Rocaglamide and a XIAP inhibitor cooperatively sensitize TRAIL-mediated apoptosis in Hodgkin's lymphomas. Int J Cancer 131: 1003-1008, 2012.

15. Luan Z, He Y, Alattar M, Chen Z and He F: Targeting the prohibitin scaffold-CRAF kinase interaction in RAS-ERK-driven pancreatic ductal adenocarcinoma. Mol Cancer 13:38, 2014.

16. Kunzi-Rapp K, Genze F, Küfer R, Reich E, Hautmann RE and Gschwend JE: Chorioallantoic membrane assay: vascularized 3-dimensional cell culture system for human prostate cancer cells as an animal substitute model. J Urol 166: 1502-1507, 2001. 
17. Chen Q, Lou W, Shen J, Ma L, Yang Z, Liu L, Luo J and Qian C: Potent antitumor activity in experimental hepatocellular carcinoma by adenovirus-mediated coexpression of TRAIL and shRNA against COX-2. Clin Cancer Res 16: 3696-3705, 2010.

18. Jin Z, Li Y, Pitti R, Lawrence D, Pham VC, Lill JR and Ashkenazi A: Cullin3-based polyubiquitination and p62-dependent aggregation of caspase-8 mediate extrinsic apoptosis signaling. Cell 137: 721-735, 2009.

19. Häcker S, Dittrich A, Mohr A, Schweitzer T, Rutkowski S, Krauss J, Debatin KM and Fulda S: Histone deacetylase inhibitors cooperate with IFN-gamma to restore caspase-8 expression and overcome TRAIL resistance in cancers with silencing of caspase-8. Oncogene 28: 3097-3110, 2009.

20. Unterkircher T, Cristofanon S, Vellanki SH, Nonnenmacher L, Karpel-Massler G, Wirtz CR, Debatin KM and Fulda S: Bortezomib primes glioblastoma, including glioblastoma stem cells, for TRAIL by increasing tBid stability and mitochondrial apoptosis. Clin Cancer Res 17: 4019-4030, 2011.

21. Sadlish H, Galicia-Vazquez G, Paris CG, Aust T, Bhullar B, Chang L, Helliwell SB, Hoepfner D, Knapp B, Riedl R, Roggo S, Schuierer S, Studer C, Porco JA Jr, Pelletier J and Movva NR: Evidence for a functionally relevant rocaglamide binding site on the eIF4A-RNA complex. ACS Chem Biol 8: $1519-1527,2013$.

22. Irmler M, Thome M, Hahne M, Schneider P, Hofmann K, Steiner V, Bodmer JL, Schröter M, Burns K, Mattmann C, Rimoldi D, French LE and Tschopp J: Inhibition of death receptors signals by cellular FLIP. Nature 338: 190-195, 1997.

23. Kim JY, Kim EH, Park SS, Lim JH, Kwon TK and Choi KS: Quercetin sensitizes human hepatoma cells to TRAIL-induced apoptosis via Sp1-mediated DR5 up-regulation and proteasome-mediated c-FLIPS down-regulation. J Cell Biochem 105: 1386-1398, 2008.

24. Bao Q and Shi Y: Apoptosome: a platform for the activation of initiator caspases. Cell Death Differ 14: 56-65, 2007.

25. Wang G, Wang X, Yu H, Wei S, Williams N, Holmes DL, Halfmann R, Naidoo J, Wang L, Li L, Chen S, Harran P, Lei X and Wang X: Small-molecule activation of the TRAIL receptor DR5 in human cancer cells. Nat Chem Biol 9: 84-89, 2013

26. Smyth MJ, Cretney E, Takeda K, Wiltrout RH, Sedger LM, Kayagaki N, Yagita $\mathrm{H}$ and Okumura K: Tumor necrosis factor-related apoptosis-inducing ligand (TRAIL) contributes to interferon gamma-dependent natural killer cell protection from tumor metastasis. J Exp Med 193: 661-670, 2001.

27. Hayakawa Y, Screpanti V, Yagita H, Grandien A, Ljunggren HG, Smyth MJ and Chambers BJ: NK cell TRAIL eliminates immature dendritic cells in vivo and limits dendritic cell vaccination efficacy. J Immunol 172: 123-129, 2004

28. Villanueva A, Hernandez-Gea V and Llovet JM: Medical therapies for hepatocellular carcinoma: a critical view of the evidence. Nat Rev Gastroenterol Hepatol 10: 34-42, 2013.
29. Nojiri K, Sugimoto K, Shiraki K, Tameda M, Inagaki Y, Ogura S, Kasai C, Kusagawa S, Yoneda M, Yamamoto N, Takei Y, Nobori T and Ito M: Sorafenib and TRAIL have synergistic effect on hepatocellular carcinoma. Int J Oncol 42:101-108, 2013.

30. Schattenberg JM, Schuchmann M and Galle PR: Cell death and hepatocarcinogenesis: Dysregulation of apoptosis signaling pathways. J Gastroenterol Hepatol 26: 213-219, 2011

31. Wang Z, Zhou X, Li J, Liu X, Chen Z, Shen G, Guan T, Ye N, Wei X, Huang N, Yang L, Wei Y and Li J: Suppression of hepatoma tumor growth by systemic administration of the phytotoxin gelonin driven by the survivin promoter. Neoplasma 60: 469-479, 2013.

32. Kelley SK and Ashkenazi A: Targeting death receptors in cancer with Apo2L/TRAIL. Curr Opin Pharmacol 4: 333-339, 2004.

33. Sayers TJ, Brooks AD, Koh CY, Ma W, Seki N, Raziuddin A, Blazar BR, Zhang X, Elliott PJ and Murphy WJ. The proteasome inhibitor PS-341 sensitizes neoplastic cells to TRAIL-mediated apoptosis by reducing levels of c-FLIP. Blood 102: 303-310, 2003.

34. Ganten TM, Haas TL, Sykora J, Stahl H, Sprick MR, Fas SC, Krueger A, Weigand MA, Grosse-Wilde A, Stremmel W, Krammer PH and Walczak H: Enhanced caspase-8 recruitment to and activation at the DISC is critical for sensitization of human hepatocellular carcinoma cells to TRAIL-induced apoptotic by chemotherapeutic drugs. Cell Death Differ 11 (Suppl 1) S86-S96, 2004

35. Qi L, Bellail AC, Rossi MR, Zhang Z, Pang H, Hunter S, Cohen C, Moreno CS, Olson JJ, Li S and Hao C: Heterogeneity of primary glioblastoma cells in the expression of caspase- 8 and the response to TRAIL-induced apoptosis. Apoptosis 16: 1150-1164, 2011.

36. Kaminskyy VO, Surova OV, Vaculova A and Zhivotovsky B: Combined inhibition of DNA methyltransferase and histone deacetylase restores caspase-8 expression and sensitizes SCLC cells to TRAIL. Carcinogenesis 32: 1450-1458, 2011.

37. Liu L, Yim H, Choi JH, Kim ST, Jin Y and Lee SK: ATM kinase promotes both caspase- 8 and caspase-9 activation during TNF- $\alpha$-induced apoptosis of HeLa cells. FEBS Lett 588: 929-935, 2014.

38. Feoktistova M, Geserick P, Kellert B, Dimitrova DP, Langlais C, Hupe M, Cain K, MacFarlane M, Häcker G and Leverkus M: cIAPs block Ripoptosome formation, a RIP1/caspase- 8 containing intracellular cell death complex differentially regulated by cFLIP isoforms. Mol Cell 43: 449-463, 2011.

39. Safa AR, Day TW and Wu CH: Cellular FLICE-like inhibitory protein (c-FLIP): a novel target for cancer therapy. Curr Cancer Drug Targets 8: 37-46, 2008.

40. Kataoka T: The caspase- 8 modulator c-FLIP. Crit Rev Immunol 25: 31-58, 2005

41. Cheung HH, Mahoney DJ, Lacasse EC and Korneluk RG Down-regulation of c-FLIP enhances death of cancer cells by smac mimetic compound. Cancer Res 69: 7729-7738, 2009.

42. Shirley S and Micheau O: Targeting c-FLIP in cancer. Cancer Lett 332: 141-150, 2013. 